\title{
Análise e Comparação da Hierarquia de Massas dos neutrinos e suas consequências no experimento DUNE.
}

\section{Ricardo O. Pinto*, Pedro C. Holanda.}

\section{Resumo}

Um dos grandes modelos da Física de Partículas é o Modelo Padrão, onde descreve três interações fundamentais do universo, e também classifica todas as partículas elementares conhecidas. Porém existem partículas, como o neutrino, que por apresenterem características e efeitos como a oscilação de neutrinos, fazem com que o modelo tenha que ser repensado ou substituído. Nesse cenário, está sendo construído o DUNE (Deep Underground Neutrino Experiment), para analisar tais efeitos e características. Através do estudo prévio de mecânica quântica, e posteriormente da análise artigos de colaboração do DUNE tal assunto é abordado.

\section{Palavras-chave:}

Oscilação de Neutrinos, Hierarquia de Massas, DUNE.

\section{Introdução}

Existem três tipos de neutrinos (e seus respectivos antineutrinos) com sabores $v_{\alpha}$ (com $\alpha=e, \mu, \tau$ ), onde estes são combinações lineares de autoestados de massa $v_{i}(i=1,2,3): v_{\alpha}=\sum U_{\alpha, i}^{t} v_{i}$, onde $U$ é a matriz de mistura. Nas oscilações de neutrinos o parâmetro relevante para a pesquisa é a diferença de massas quadrada $\Delta m_{j i}^{2}=m_{j}^{2}-m_{i}^{2}$, essa é exatamente a medida a qual esse estudo se baseia, pois com a determinação das diferenças de massas, ou seja, com a Hierarquia de Massas(HM) bem determinada, é possível descrever a matriz Hamiltoniana de massas $\left(H_{\text {mass }}\right)$. Com isso é possível obter a matriz $H_{\text {flavour }}=U\left(H_{\text {mass }}\right) U^{t}+V$, que faz com que parte da equação de Schrodinger que descreve a evolução dos estados do sistema seja determinada. No caso a equação de Schrödinger é dada por: $i \frac{\partial}{\partial t} v^{(\alpha)}=\left[H_{\text {flavour }}\right] v^{(i)}$. A matriz de mistura $U$ é dependente dos ângulos de mistura na oscilação de neutrinos, que também são objetos de pesquisa no experimento DUNE. O objetivo deste estudo é, além de estudar mecânica quântica voltada para o caso, analisar a perspectiva de onde se encontra a discussão da HM, assim como analisar artigos colaborativos do futuro experimento a respeito do assunto abordado.

\section{Resultados e Discussão}

Hoje já é de conhecimento que $m_{2}^{2}>m_{1}^{2}$, $\Delta m_{21}^{2} \simeq 7.6 \times 10^{-5} \mathrm{eV}^{2}$ e que $\Delta m_{32}^{2} \simeq 2.5 \times 10^{-3} \mathrm{eV}^{2}$ [1], mas ainda não se sabe se $m_{2}$ é mais leve ou mais pesada que $m_{3}$. No primeiro caso (em que $m_{2}$ é mais leve) dizemos que a HM é normal e no segundo caso que a HM é invertida. Como o Hamiltoniano na base de massa $\left(H_{\text {mass }}\right.$ ) depende apenas de $\Delta m_{21}^{2}, \Delta m_{32}^{2}$ e $E_{v}$ (energia dos neutrinos envolvidos no processo), fica possível determinar essa matriz Hamiltoniana. A partir de artigos de colaboração do experimento DUNE, também foi possível analisar a sensibilidade esperada do experimento em relação à $\mathrm{HM}$ :
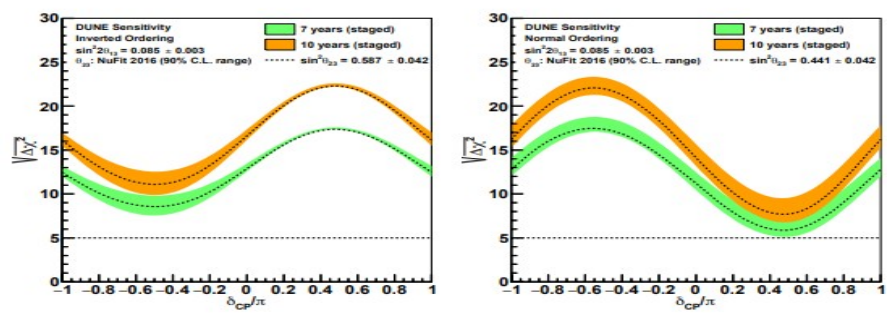

Figura 1. Sensibilidade do DUNE para HM como função do verdadeiro valor de $\delta$. No gráfico da direita (esquerda) HM normal (invertida) assumida [2].

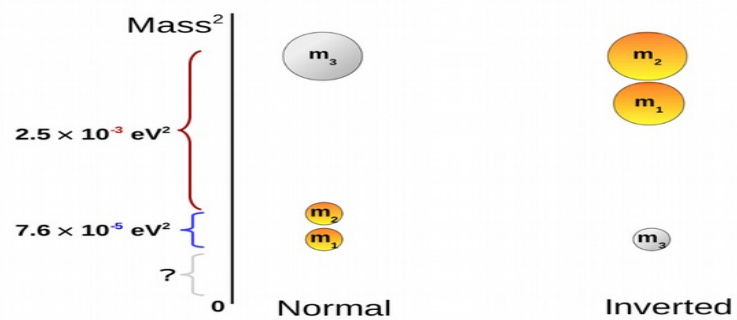

Figura 2. Imagem ilustrativa da Hierarquia de massas dos Neutrinos (site: http://www.hyper-k.org/en/physics/img/hierarchy sp heres english.png, 14/07/2018, 20h).

\section{Conclusão}

A determinação da HM pode ser um importante passo para mostrar se o neutrino é sua própria antipartícula. Pode-se dizer que $v_{\mu}$ e $v_{2}$, por exemplo, são semelhantes a respeito do conceito de partícula, eles tem apenas propriedades diferentes. Pode-se interagir com os neutrinos $v_{\alpha}$ mas não temos informação sobre suas massas, contudo não podemos interagir diretamente com $v_{i}$ mas estes têm massas bem definidas.

${ }^{1}$ R. Acciarri et al. [DUNE Collaboration], [arXiv:1512.06148].

${ }^{2}$ D. Brailsford [DUNE Collaboration], arXiv:1804.04979 [physics.ins-det].

${ }^{3}$ Thomson, Mark. Modern Particle Physics. Cambridge University Press, 2013. 546 p. 